\title{
DURABILIDAD DE LA MADERA DEL Pinus taeda L. IMPREGNADO CON ARSENIATO DE COBRE CROMATADO, FRENTE A HONGOS XILÓFAGOS, MISIONES, ARGENTINA
}

\author{
Elisa Alicia Bobadilla*, Teresa María Suirezs *, Obdulio Peryra*, Elizabeth Weber*, \\ Fidelina Silva*, Alicia Mónica Stehr ** \\ *Ing. Forestal, M.Sc., Facultad de Ciencias Forestales, UNaM, Misiones, Argentina - elisa@facfor.unam.edu.ar - \\ bernio@ceel.com.ar - opereyra@facfor.unam.edu.ar - eweber@facfor.unam.edu.ar - fidelina@facfor.unam.edu.ar \\ **Ing. Forestal, Facultad de Ciencias Forestales, UNaM, Misiones, Argentina - astehr@facfor.unam.edu.ar \\ Recebido para publicação: 19/05/2006 - Aceito para publicação: 16/08/2006
}

\begin{abstract}
Resumen
El objetivo del trabajo fue estudiar el comportamiento de la madera de Pinus taeda L. sin impregnar e impregnada con CCA tipo C, con retenciones de 5,10 y $15 \mathrm{~kg} / \mathrm{m}^{3}$, frente a la acción de los hongos xilófagos, Pycnoporus sanguineus, Laetiporus sulphureus, Plerotus sajor caju, Ganoderma applanatum, Glophyllum sp. en ensayos de laboratorio. Las maderas fueron obtenidas de seis árboles seleccionados al azar, e impregnadas con CCA en una autoclave de laboratorio. Para determinar la eficacia del preservante fueron utilizadas, 5 probetas de $3 \times 1 \times 0,5 \mathrm{~cm}$ por retención y por cepa de hongos y 5 de madera sin impregnar, testigo. Las probetass fueron puestas en ambiente de laboratorio para climatización y fueron pesadas. Los hongos fueron cultivados en tubos de ensayos de $20 \mathrm{~mm}$ de diámetro y $200 \mathrm{~mm}$ de largo, y sobre los mismos se colocaron las probetas, permaneciendo en estufa a $28^{\circ} \mathrm{C}$ por 12 semanas. Luego de este período las probetas fueron retiradas y puestas en ambiente de laboratorio, hasta obtener el contenido de humedad del inicio del ensayo. La degradación fue evaluada a través de la pérdida de peso. La madera de Pinus taeda L. fue moderadamente resistente con retenciones de 5 y $10 \mathrm{~kg} / \mathrm{m}^{3}$ de CCA, por lo tanto la retención de $5 \mathrm{~kg} / \mathrm{m}^{3}$ es suficiente.

Palabras claves: Pinus taeda; CCA; durabilidad; hongos xilófagos.
\end{abstract}

\begin{abstract}
Durability of Pinus taeda L. wood, impregnated with chromated copper arsenate to the xylophagaus fungi action. Misiones, Argentina. The objective of this work was to study the behavior of Pinus taeda L. wood without and with impregnation with retentions of 5,10 and $15 \mathrm{~kg} / \mathrm{m}^{3}$ of chromated copper arsenate, type C, preservative against Pycnoporus sanguineus, Laetiporus sulphureus, Plerotus sajor caju, Ganoderma applanatum, Glophyllum sp. in laboratory conditions. The wood was obtained from six trees chosen at random, and impregnated with C.C.A. in laboratory autoclave. To determine the efficiency of preservative 5 samples were used 5 samples of $3 \times 1 \times 0,5 \mathrm{~cm}$ of no treated wood, were used. The wood samples were sterilized and put on a xylophagaus fungi staying in oven at $28^{\circ} \mathrm{C}$ for 12 weeks; after this period, the samples were taken away and put on a laboratory environment, until the moisture content of the beginning of the experiment was attained. The degradation was evaluated through the weight loss. The Pinus taeda L. wood was moderately resistant with retentions of 5 and $10 \mathrm{~kg} / \mathrm{m}^{3}$ of CCA, therefore retention of $5 \mathrm{~kg} / \mathrm{m}^{3}$ is enough.

Keywords: Pinus taeda; CCA; durability; xylophagaus fungi.
\end{abstract}

\section{INTRODUCCIÓN}

En la Argentina el Pinus taeda L. se encuentra ampliamente difundido en las reforestaciones de las provincias de Misiones, Corrientes y en menor escala en Córdoba. Esta especie comenzó a tener importancia en cuanto a superficies anuales cultivadas a mediados de la década del 1970, considerando su participación en aproximadamente $50 \%$ de las 260.000 hectáreas existentes ((GONZALEZ et al.,1992).

Actualmente en Misiones la tendencia es el cultivo del Pinus taeda por su rápido crecimiento, esta especie con plantines de origen seleccionados pueden llegar a un crecimiento de más de 40 $\mathrm{m}^{3} /$ ha/año. Se lo cultiva con densidades de 2.000, 1.600 y 1.100 plantines/ha. (ARGENTINA, 1999). 
Los productos de los raleos son utilizados en la industria de pasta celulósica, paneles aglomerados y tableros de fibras tipo MDF. Los rollizos de mayor diámetro para aserrío, empleándose en carpintería de obra, revestimiento de interiores, tarimas livianas, envases, cajonería y en la industria del debobinado para la fabricación de maderas compensadas (LEONARDIS, 1977).

La madera de Pinus taeda es susceptible al ataque de hongos en algunas condiciones de uso, principalmente en lugares húmedos y en contacto con el suelo. Los hongos que atacan a las maderas se clasifican según los daños que producen, en pudriciones, manchas y mohos. Las pudriciones son producidas por hongos basidiomicetes que desintegra a la lignina por oxidación y a la celulosa por hidrólisis, causando pérdida del color natural, peso y propiedades de resistencia mecánica de la madera (MARTÍNEZ, 1952).

El uso de la madera impregnada está poco difundida en la Argentina. La proporción de la misma en la producción de pino no llega al 5\% (PELAGALLO, 1997). Las maderas impregnadas con hidrosolubles pueden ser usadas en las construcciones exteriores de las viviendas, en cercos, plantaciones de vid, postes, además en la construcción de vigas multilaminadas, apreciándose un aumento en su utilización.

Las maderas tratadas con productos químicos no deben ser cepilladas ni perforadas, con el fin de evitar cualquier tipo de contaminación ambiental; por ese motivo se recomienda realizar todo tipo de taladrado, perforado y otros cortes antes del impregnado. (También se expone la madera no tratada al ataque)

Según Tinto (1980), las maderas tratadas con preservantes hidrosolubles no presentan inconvenientes en la aplicación de pinturas, barnices y lustres siempre que el material esté seco. La incorporación de productos preservantes en la masa leñosa afecta sus propiedades en sentido positivo, dándole mayor resistencia frente al ataque de los agentes destructores, pero a veces aparecen reacciones negativas, esto depende del tratamiento y la concentración del producto como la reducción de sus resistencias mecánicas, porque la madera se vuelve quebradiza.

Las maderas impregnadas con el hidrosoluble arseniato de cobre cromatado (CCA) se tornan verdosas y cuanto mayor es la retención del producto adquieren un color más oscuro.

Una vez impregnadas las maderas, estas deben estacionarse como mínimo siete días a $25^{\circ} \mathrm{C}$ para que se produzcan todas las reacciones químicas, logrando su fijación dentro de la madera, por lo tanto deben estar protegidas de la lluvia en ese intervalo de tiempo, evitando de esta manera que se diluya el producto que se encuentra en la superficie antes de su fijación. Después de seca, la misma puede ser manejable sin riesgo para el hombre y los animales domésticos (WILKINSON, 1979).

Un documento citado en "Britsh Wood Preserving Association Treatment Specifications State" en 1975 dice que la madera tratada con CCA logra una vida útil de 15, 30 y hasta 40 años en servicio, como ser torres de enfriamiento, muelles y postes (WILKINSON, 1979).

El CCA es un producto hidrosoluble que combina el efecto fungicida del cobre con el insecticida del arsénico y una sal alcalina de cromo con el fin de obtener una alta fijación de los principios activos de los componentes mencionados (TUSET Y DURAN, 1979). Según Fahlstrom (1978), el CCA se ha comenzado a usar desde 1930 y su aplicación ha sido creciente desde entonces. Su formulación ha cambiado. Se lo puede obtener en forma de sales cristalinas, pastas o líquidos concentrados.

El "American Word Preservers Asociation" (AWPA, 1996), establece tres tipos de formulaciones distintas del CCA, clasificados según el porcentaje de óxidos en su composición en A, B y C presentados en la tabla 1.

Tabla 1. Clasificación del CCA según sus \% de óxidos que lo componen.

Table 1. Classification of CCA according to its \% of oxides that compose it.

\begin{tabular}{lccc}
\hline \multirow{2}{*}{ Componentes } & \multicolumn{3}{c}{ Tipos de CCA (\%) } \\
\cline { 2 - 4 } & A & B & C \\
\hline $\mathrm{Cr} \mathrm{O}_{3}$ & 65,5 & 35,3 & 47,5 \\
$\mathrm{Cu} \mathrm{O}$ & 18,1 & 19,6 & 18,5 \\
$\mathrm{As}_{2} \mathrm{O}_{5}$ & 16,4 & 45,1 & 34,0 \\
\hline
\end{tabular}

Esta clasificación fue establecida por AWPA (1996) después de varios experimentos realizado por Smith y Williams, quienes verificaron que la máxima eficiencia no coincide con la máxima fijación de cobre y arsénico (LEPAGE, 1986). 
Según Normas del Instituto Argentino de Racionalización de Materiales - IRAM 9600 (1992), los valores nominales mínimos de retención para maderas de coníferas, en zonas donde hay más riesgos de termitas las retenciones deben ser de $4 \mathrm{~kg} / \mathrm{m}^{3}$ de óxidos para maderas sin contacto con el suelo; y 6,4 $\mathrm{kg} / \mathrm{m}^{3}$ para maderas en contacto con el suelo. En zonas sin riesgo las retenciones para coníferas son de 5 $\mathrm{kg} / \mathrm{m}^{3}$ y $8,5 \mathrm{~kg} / \mathrm{m}^{3}$ en contacto con el suelo.

Según Norma AWPA (1996), para lograr una protección efectiva de la madera se requiere una retención neta mínima de óxidos de CCA de 6 a $9,6 \mathrm{~kg} / \mathrm{m}^{3}$ y recomienda, según el uso final de las maderas, las siguientes retenciones mínimas de componentes activos (óxidos), son menores para madera de uso exterior sin contacto con el suelo, en condiciones secas o húmedas, retenciones de $6 \mathrm{~kg} / \mathrm{m}^{3}$ para madera de uso exterior, en contacto directo con el suelo se requiere una retención de 8 a $12 \mathrm{~kg} / \mathrm{m}^{3}$, para maderas en torres de enfriamiento se requiere una retención de 10 a $16 \mathrm{~kg} / \mathrm{m}^{3}$.

Según Pizzi et al. (1984), al reaccionar el CCA con la madera, se produce una disminución del $\mathrm{pH}$, cuya magnitud depende de la concentración del producto. Se le atribuye esa baja a la fijación del cobre por cambio iónico con liberación de protones. Después de esta disminución instantánea del pH comienza a aumentar con la formación de los complejos lignino-ácido crómicos o lignina-cromato de cobre y también se produce la reducción del cromo. La mayor parte del cobre se fija en la capa S1 de la pared celular, reaccionando con la lignina en un 80 a $90 \%$ y otra parte reacciona con la celulosa.

El objetivo de este trabajo consistió en determinar el comportamiento de la madera de Pinus taeda L. sin impregnar e impregnada con retenciones de 5,10 y $15 \mathrm{~kg} / \mathrm{m} 3$, con preservador arseniato de cobre cromatado, tipo C, frente a la acción de los hongos xilófagos, Pycnoporus sanguineus, Laetiporus sulphureus, Pleurotus sajor caju, Ganoderma applanatum, Glophyllum sp. en ensayos de laboratorio.

\section{MATERIAL Y MÉTODOS}

El material utilizado para la confección de las probetas de madera, ensayadas en este trabajo, fueron obtenidas de 6 árboles de Pinus taeda L. provenientes de una plantación de 14 años de edad en un suelo rojo profundo, complejo 9, situado en el Municipio de Caraguatay, Departamento Montecarlo en la Provincia de Misiones, Argentina. Una vez apeados, se seccionaron toras desde la base hasta 1,20 m del fuste denominándose como tora $\mathrm{A}$, y de $1,20 \mathrm{~m}$ a $2,40 \mathrm{~m}$ como tora $\mathrm{B}$. En los extremos de cada una de ellas se demarcaron cuatro viguetas de sección cuadrada de $7 \times 7 \mathrm{~cm}$, indicando el número de árbol, denominación y ubicación (N - S - E - O) de las mismas.

Las maderas fueron impregnadas por el método de Burnett con el preservante CCA en equipo de laboratorio a tres concentraciones distintas, tales que permitieran retenciones de 5,10 y $15 \mathrm{~kg} / \mathrm{m}^{3}$. Previo a dichas impregnaciones, se midieron los volúmenes y pesos de las piezas de maderas, con el fin de corroborar la retención de cada una de ellas y fueron estacionadas hasta adquirir un contenido de humedad del $12 \%$. Luego se prepararon las probetas siguiendo las Normas IRAM 9518, (1962). Las dimensiones de las probetas fueron de $3 \times 1 \times 0,5 \mathrm{~cm}$, el número de probetas fue de 5 unidades por tratamiento y por cepa de hongos, que se nombran a continuación, Pycnoporus sanguineus - BAFC 2341, Laetiporus sulphureus o Polyporus sulphureus - BAFC 205, Ganoderma applanatum - BAFC 1168, Gloeophyllum sp.- BAFC 2735 y Plerotus sajon caju. Estas cepas de hongos fueron provistas por el Departamento de Ciencias Biológicas de la Facultad de Ciencias Exactas y Naturales de la Universidad de Buenos Aires (UBA).

Las probetas fueron pesadas y colocadas en un desecador con agua destilada, de modo que actúe como cámara húmeda, luego fueron llevadas a estufa a $110^{\circ} \mathrm{C}$, durante $1 / 2$ hora para su esterilización; posteriormente se disminuyó la temperatura a $30{ }^{\circ} \mathrm{C}$ y se mantuvo durante 12 horas, de modo que alcancen una humedad suficiente que se estima en $30 \%$ de agua contenida.

Se utilizó como medio de cultivo para las cepas 25 gr de agar y $15 \mathrm{gr}$ de extracto de malta por cada $1000 \mathrm{ml}$ de agua destilada, (IRAM 9518, 1962); luego se colocó en tubos Pirex de $200 \mathrm{~mm}$ de largo y $20 \mathrm{~mm}$ de diámetro, obturados con tapón de algodón. Todo este material fue esterilizado en autoclave a $0,5 \mathrm{~atm}$. de presión durante 30 minutos.

Las cepas se cultivaron durante 14 días en cajas de Petri con el medio indicado, manteniéndolas a $27{ }^{\circ} \mathrm{C} \pm 1{ }^{\circ} \mathrm{C}$ en estufa de cultivo. Luego en condiciones asépticas se colocó en cada tubo pequeños trozos de micelios, los cuales se dejaron 14 días en las mismas condiciones ya descriptas, hasta que el crecimiento del micelio cubrió totalmente el pico de flauta; posteriormente se pusieron las probetas antes 
mencionadas en contacto con el hongo. Cada tubo fue identificado adecuadamente y depositado en una estufa de cultivo durante tres meses.

Las probetas se controlaron semanalmente, para observar si algunas de las muestras presentaban contaminación o el hongo no se desarrollaba normalmente. A los tres meses se dio por finalizado el ensayo, se extrajeron las probetas, se retiró el micelio remanente en las mismas y se estacionaron en ambiente de laboratorio durante tres días. Posteriormente se tomó el peso de cada probeta. El \% de pérdida de tejido leñoso por la acción del hongo se determinó por diferencia de peso inicial y final.

De acuerdo a esta pérdida de peso se las puede clasificar siguiendo el criterio de Findlay, (1938), quien clasificó las maderas en 5 grupos, basado en que los promedios de pérdidas de peso producido por los hongos durante un determinado lapso de tiempo, estaban relacionadas con su grado de resistencia a la pudrición. Dicha clasificación se presenta en la tabla 2:

Tabla 2. Relación del porcentaje de pérdida de peso y grado de resistencia.

Table 2. Ralation of percent of weight lost and degree resistance.

\begin{tabular}{lc}
\hline Porcentaje de pérdida de peso & Grado de durabilidad \\
\hline$<1 \%$ & muy resistente \\
$2-5 \%$ & Resistente \\
$5-10 \%$ & Moderadamente resistente \\
$10-30 \%$ & no resistente \\
$>$ al $30 \%$ & Perecederas \\
\hline
\end{tabular}

\section{RESULTADOS Y DISCUSION}

En la tabla 3 se denota la diferencia estadísticamente significativa de los tratamientos efectuados.

Tabla 3. Análisis de varianza.

Table 3. Variance analysis.

\begin{tabular}{lcccc}
\hline Fuente de variación & Grados de libertad & Suma de cuadrados & Cuadrado medio & F \\
\hline Tratamiento & 3 & 28480,71 & 9493,57 & $120,19^{* *}$ \\
Error & 96 & 7582,08 & 78,98 & \\
Total & 99 & 36062,79 & & \\
\hline
\end{tabular}

** Significativo a $1 \%$ de probabilidad

En la tabla 4 se presenta la comparación entre porcentaje de pérdida de peso medio de maderas tratadas con 5,10 y $15 \mathrm{~kg} / \mathrm{m}^{3}$ de CCA y madera sin tratar. Entre las maderas tratadas no se observaron diferencias estadísticamente significativas, sí entre estas y la madera sin tratar.

Tabla 4. Comparación de medias para las maderas tratadas y no tratadas.

Table 4. Comparison of means of treated and not treated woods.

\begin{tabular}{lc}
\hline Retención $\mathbf{( k g / \mathbf { m } ^ { 3 } )}$ & Media (\% pérdida) \\
\hline 15 & $5,42 \mathrm{a}$ \\
5 & $8,87 \mathrm{a}$ \\
10 & $9,31 \mathrm{a}$ \\
Testigo & $46,69 \mathrm{~b}$ \\
\hline
\end{tabular}

Valores medios seguidos de igual letra indican que no hay diferencias significativas ( Tukey, $\mathrm{p}>0,05$ ).

En la tabla 5 quedan demostradas las diferencias entre las maderas tratadas con retenciones de 5 y $10 \mathrm{~kg} / \mathrm{m}^{3}$, respecto a las maderas tratadas con retención de $15 \mathrm{~kg} / \mathrm{m}^{3}$. Entre las maderas con retenciones de $5 \mathrm{~kg} / \mathrm{m}^{3}$ y $10 \mathrm{~kg} / \mathrm{m}^{3}$ no hubieron diferencias significativas frente a la acción de los hongos bajo estudio.

En la tabla 6 se indica una pérdida de peso para las maderas con retenciones de 5 y $10 \mathrm{~kg} / \mathrm{m}^{3}$, con respecto a todos los hongos bajo estudio, de alrededor del $9 \%$; según la clasificación de Findlay (1938) corresponde a una madera moderadamente resistente. La madera con retención de $15 \mathrm{~kg} / \mathrm{m}^{3}$ corresponde 
en promedio, para todas las especies de hongos, a un porcentaje de no más de $5 \%$ de pérdida de peso, lo cual indica que es una madera resistente al ataque frente a los tres hongos bajo estudio.

Tabla 5. Comparación de medias entre tratamientos.

Table 5. Comparison of means betwen treatments.

\begin{tabular}{lc}
\hline Retención $\left(\mathbf{k g} / \mathbf{m}^{\mathbf{3}}\right)$ & Media (\% pérdida) \\
\hline 15 & $5,42 \mathrm{~b}$ \\
5 & $8,87 \mathrm{a}$ \\
10 & $9,31 \mathrm{a}$ \\
\hline \multicolumn{2}{l}{ Valores medios seguidos de igual letra indican que no hay diferencias significativas (Tukey, $\mathrm{p}>0,05)$}
\end{tabular}

La última columna demuestra la susceptibilidad al ataque de los hongos de la madera de Pinus taeda sin ningún tratamiento (testigo), y de acuerdo a la pérdida de peso se lo clasifica como madera perecedera.

Es importante resaltar en los resultados que cuando la madera está tratada independientemente de la retención, la acción de los diferentes hongos no demuestra diferencias significativas entre ellos. Todavía se puede demostrar que la acción de los hongos en madera sin tratamiento es significativa.

Tabla 6. Porcentaje de pérdida de peso en función de la retención de CCA en $\mathrm{kg} / \mathrm{m} 3$, por especie de hongo.

Table 6. Percentage of weight loss in function of CCA retention in $\mathrm{kg} / \mathrm{m} 3$, per fungus species.

\begin{tabular}{|c|c|c|c|c|}
\hline \multirow{2}{*}{ Especies de hongos } & \multicolumn{4}{|c|}{ \% Pérdida de peso en función de la retención } \\
\hline & $5 \mathrm{~kg} / \mathrm{m}^{3}$ & $10 \mathrm{~kg} / \mathrm{m}^{3}$ & $15 \mathrm{~kg} / \mathrm{m}^{3}$ & Testigo \\
\hline Pycnoporus sanguineus BAFC: 2341 & 10,20 & 9,51 & 5,60 & 34,63 \\
\hline Ganoderma applanatum BAFC: 1168 & 8,66 & 9,71 & 5,65 & 36,77 \\
\hline Pleurotus sajor caja & 8,04 & 9,39 & 5,66 & 31,07 \\
\hline Gloeophyllum sp BAFC: 2735 & 9,08 & 9,47 & 5,45 & 63,36 \\
\hline Laetiporus sulphureus BAFC: 205 & 8,41 & 8,49 & 4,78 & 67,63 \\
\hline
\end{tabular}

\section{CONCLUSION}

La madera de Pinus taeda L. fue perecedera sin impregnar, moderadamente resistente con retenciones de 5 y $10 \mathrm{~kg} / \mathrm{m}^{3}$ de CCA, y resistente frente a la acción de las cinco especies de hongos cuando presenta una retención de $15 \mathrm{~kg} / \mathrm{m}^{3}$ de CCA.

\section{REFERENCIAS}

AMERICAN WOOD PRESERVERS ASSOCIATION. Standard P5/86: Standards for waterborne Preservatives. Stevensville, 1996.

ARGENTINA. Ministerio DE ECOLOGIA Y R. N. R. Anales de la Administración Nacional de Bosques. Buenos Aires, 1999. 179p.

FAHLSTROM, G. B. Copfer-Chrome-Arsenate Wood preservatives a study of the influence of composition on service perfomance. In: AMERICAN WOOD PRESERVERS ASSOCIATION. Proceedings of... [S.l.: s.n.], 1978. p.6.

FINDLAY, W. P. K. The value of laboratory test on Word preservative. [S.1.]:Convention British Word Preserving Asosiation . 1938

GONZALEZ, R. A.; PEREYRA, O.; SUIREZS, T. M. "Propiedades Físicas y Mecánicas de la madera de Pinus elliottii reforestado en la provincia de Misiones, Argentina". Yvyraretá, Eldorado, n. 3, p. 5-19, 1992. 
INSTITUTO ARGENTINO DE RACIONALIZACIÓN DE MATERIALES. IRAM 9518. Buenos Aires, 1962.

INSTITUTO ARGENTINO DE RACIONALIZACIÓN DE MATERIALES. IRAM 9600. Buenos Aires, 1992.

LEONARDIS J. Esencias forestales no autóctonas cultivadas en la Argentina de aplicación ornamental y/o industrial. [Buenos Aires]: Celulosa Argentina, 1977. (Libro del Árbol, t.3).

LEPAGE, E. S. Manual de preservação de madeiras: volumen I. São Paulo: Instituto de Pesquisas Tecnológicas, 1996. p.1-7.

MARTINEZ, J. B. Conservación de madera en sus aspectos teóricos, industrial y económico: volumen I. Madrid: Ministerio de Agricultura. Instituto forestal de investigaciones y experiencias, 1952. $550 \mathrm{p}$.

PELAGALLO, A. La impregnación ofrece seguridad para usar madera en la construcción. Revista Asora Argentina, n. 11, p. 38- 40, 1997.

PIZZI, A.; OROVAN, E.; SINGMIN, M.; JANSEN, A.; VOGEL, M. C. Experimental variations in the distribution of CCA preservative in lignin and holocellulose as a function of treating conditions (temperature, concentration, Ph, species and time). Stockholm: The International Research Group on Wood Preservation. 1984. p. 22. Working Group III: Preservatives and methods of treatament.

TINTO, J. C. Manual para tratamieto protectores de productos forestales. Consejo General de inversiones. San Martín 871- Capital Federal - Argentina. Pg. 62. 1980.

TUSET, R.; DURAN, F. Manual de maderas comerciales, equipos y procesos de utilización. Editorial Agropecuaria, Hemisferio Sur S.R.L. Montevideo.1979.

WILKINSON, J. G. Industrial Timber preservation. London: Associated business, 1979. 532p.

London. Pg.532. 1979. 